\title{
Why value sensitive design needs ethical commitments
}

\author{
Naomi Jacobs ${ }^{1} \oplus$. Alina Huldtgren ${ }^{2}$
}

Published online: 12 July 2018

(c) The Author(s) 2018

\begin{abstract}
Currently, value sensitive design (VSD) does not commit to a particular ethical theory. Critiques contend that without such an explicit commitment, VSD lacks a methodology for distinguishing genuine moral values from mere stakeholders-preferences and runs the risk of attending to a set of values that is unprincipled or unbounded. We argue that VSD practitioners need to complement it with an ethical theory. We argue in favour of a mid-level ethical theory to fulfil this role.
\end{abstract}

Keywords Ethical theory $\cdot$ Applied ethics $\cdot$ Value sensitive design $\cdot$ Ethics and technology

\section{Introduction}

Value sensitive design (VSD) is an approach to the design of technology "that accounts for human values in a principled and comprehensive manner throughout the design process" (Friedman et al. 2013, p. 55). The unique opportunity that the VSD approach brings to the fore is a proactive integration of ethics in the design of technology (Van den Hoven 2008).

Although VSD draws on ethical theories to identify values that are relevant for technology design (Friedman et al. 2013), VSD makes no explicit commitment to particular ethical theories. This gives rise to multiple critiques to VSD.

Manders-Huits argues that VSD cannot provide a "methodological account for distinguishing genuine moral values from mere preferences, wishes, and whims of those involved in the design process" (2010, p. 281). And without such a methodological account, VSD practitioners risk attending to an unprincipled or unbounded set of values. Albrechtslund (2007) points out that although VSD draws on ethical theory, it is not clear "what theories and which values this includes" (p. 67). Borning and Muller (2012) argue that

Naomi Jacobs

N.Jacobs@tue.nl

Alina Huldtgren

alina.huldtgren@hochschule-rhein-waal.de

1 Eindhoven University of Technology, Eindhoven, The Netherlands

2 Rhine-Waal University of Applied Sciences, Kamp-Lintfort, Germany
VSD practitioners, via the rhetorical move of using a disembodied voice, claim more authority and impartiality than is warranted in areas in which the practitioners' own normative assumptions may limit exactly those qualities of authority and impartiality.

Therefore, we argue, VSD practitioners should make use of an ethical theory complementary to the VSD method. Such an ethical theory can provide sources of justification and argumentation for moral claims and considerations, which are needed to make principled judgments, to attend to a set of bounded and principled values, and to legitimize value trade-offs during the design process.

The article proceeds as follows: first, we argue that VSD practitioners' explicit use of an ethical theory to complement the VSD process can resolve the critique that the voice and values of researchers and designers engaging in VSD is often insufficiently explicit (Borning and Muller 2012).

Secondly, we turn to the much-debated topic of whether or not VSD can rely on a set of universal values (Borning and Muller 2012; Friedman et al. 2013). We argue that by relying too much on empirical studies of universal values, VSD runs the risk of committing the naturalistic fallacy, and that an explicit commitment by VSD practitioners to ethical theory can resolve this.

Thirdly, we turn to the central question of this paper: what kind of ethical theory is best suited to accompany VSD practitioners in the VSD process? We argue for a mid-level ethical theory, and discuss several desiderata that are necessary for such a mid-level ethical theory to successfully accompany VSD. 


\section{The voice of the researchers}

Borning and Muller (2012) have pointed out that researchers and designers engaging in VSD often fail to make explicit their own part in the design process, including their values and normative commitments, and unintentionally claim (unjustified) moral authority or impartiality.

It is relevant, however, for participants in a VSD project, or readers of the VSD project results, to know what the relevant values of the researchers and designers are, as well as the values that are explicitly supported in the project. Making explicit what values underlie the development and resulting functionality of a certain technology can help to assess the design process, the future impact of a technology on its users, and help potential users to choose a technology matching their own values. The most evident way to do this is by indicating at the start of the VSD process what moral principles the practitioners are making use of and adhere to them throughout the design process.

\section{Universal values and the naturalistic fallacy}

Friedman et al. (2013) take the controversial position that certain values are universally held and that this fact can provide normative direction in design. Friedman et al. (2013) contend that this empirical proposition is grounded in a large body of psychological and anthropological data.

Whether or not this empirical claim is correct is not the crucial point here. What is crucial is that versions of VSD that are heavily informed by this notion run the risk of committing the naturalistic fallacy. Advocates of this approach implicitly assume that one will know what to do in a normative sense, once one knows empirically whether some values are universally held (Manders-Huits 2010). However, even if empirical data is able to show that people hold certain values, it is not able to say anything about whether people should hold certain values. Ethical theories can provide arguments why we should care about certain values, why certain values should be prioritized over others, and how to make value trade-offs in case of value conflicts.

We agree with Borning and Muller (2012) that VSD does not need to take a position on whether or not it is empirically true that certain values are universally held. More important is whether VSD can address questions concerning value prioritization in the case of value conflicts. We believe that listening to the voices of stakeholders is important to identify what values matter and their input may also provide directions for value trade-offs. However, as Manders-Huits (2010) points out, when the value stances of stakeholders are the normative input for the VSD analysis, then VSD risks conflating facts and values. To avoid the naturalistic fallacy, the normative input for the VSD analysis should be derived from ethical theory.

\section{Ethical theory}

What kind of ethical theory is best suited to accompany VSD? First, there are ethical theories that work with a topdown approach: general precepts are "applied" to particular cases. For instance, the principle of utility from classical utilitarian theory, or the categorical imperative from Kantian theory is applied to a practical problem. An often made critique to such top-down approaches is that the abstract principles in classical ethical theories, like e.g. the utility principle or Kant's categorical imperative, are extensively indeterminate, i.e. the content of these principles are often too abstract to determine specific acts that should (not) be performed (Beauchamp and Childress 2013). Therefore, it's doubtful whether these abstract principles from classical ethical theories "can be fruitfully "applied" directly, so as to yield univocal answers to complex problems of professional practice and public policy" (Arras 2016).

Secondly, there are bottom-up approaches in applied ethics. Such approaches emphasize that moral certitude (or the best approximation thereof) is to be found on the level of specific cases. Instead of using principles from ethical theory, one needs to look at all the particularities of a case. One then compares these details with the details of so-called paradigm cases, on which we have settled moral judgments, using analogical reasoning to make a judgment (Arras 2016).

A critique to these bottom-up, casuistic approaches is that they seem to "black-box" or obscure moral justification, making it "difficult to provide (an account of) public justification of moral judgments" (Van den Hoven 2008, p. 54). Another point of critique is that proponents of bottom-up, casuistic approaches write as if paradigm cases speak for themselves or inform moral judgements by their facts alone, however, to move constructively from case to case, a recognized and morally relevant norm must connect the cases. "All analogical reasoning in casuistry requires a connecting norm to indicate that one sequence of events is morally like or unlike another sequence in relevant respects" (Beauchamp and Childress 2013, p. 401). So understood, casuistry requires ethical principles after all in order to give analogical reasoning moral direction.

Thirdly, there are mid-level approaches that often consist of a cluster of pivotal moral principles. These principles function as an analytical framework, forming the starting point for applied ethics in a specific domain, e.g. biomedical ethics, environmental ethics, or ethics of technology. Mid-level principles function as general guidelines for the formulation of more specific rules and are usually regarded 
as generating "prima facie" obligations, i.e. as an obligation that must be fulfilled unless it conflicts with an equal or stronger obligation (Ross 1930). In order to come to concrete action-guidance, principles must be specified. The process of specification consists of reducing the indeterminacy of abstract norms by narrowing the scope, which comes down to "spelling out where, when, why, how, by what means, to whom, or by whom the action is to be done or avoided" (Richardson 1990, p. 289). When two rival but valid specifications of principles conflict with each other, then these conflicting specifications need to be balanced and weighed against each other (Beauchamp and Childress 2013).

A critique to such mid-level theories is that ranking, specification, and balancing can vary greatly among different people regarding a particular case (Gordon et al. 2011), and that it is unclear how a justified decision between opposing principles in such a case is to be made.

Although mid-level approaches are not free from criticism, we believe that mid-level theory is the right kind of ethical theory to accompany VSD practitioners. Let us explicate.

First, mid-level approaches are convergent; i.e. differences on the highest level of moral theory most often converge at the level of mid-level action guiding principles. As James Sterba puts it: "traditional ethical theories, be they Aristotelian, Kantian, Millian, or whatever, have come to be revised and reformed in such a way that, at least in their most morally defensible formulations, they no longer differ in the practical requirements they endorse" (2005, p. 1). For example, a utilitarian and a Kantian deontologist might differ at the foundational level of moral theory, these differences ultimately can recede at the level of mid-level moral principles where the utilitarian and the Kantian deontologist could agree, for instance, on the importance of the principle of respect for autonomy in a specific practice such as research ethics (Arras 2016). In a context where people with various disciplinary backgrounds, interests, and priorities have to work together, which often is the case in designcontexts, convergence on the practical level is crucial to come to joint decisions.

Secondly, mid-level principles can provide action-guidance in concrete cases, in contrast to general precepts from classical moral theories that are often too indeterminate to do so. Mid-level principles can provide action-guidance in practice because they are (a) domain specific, that is, the selection of a cluster of principles often takes place after examining considered moral judgments and the way moral beliefs cohere in a certain context (Beauchamp and Childress 2013). And (b) because through the methodological tool of specification, the indeterminacy of abstract norms is reduced and content is added to abstract principles, ridding them of their indeterminateness and providing action-guiding content for the purpose of coping with complex cases (Beauchamp and Childress 2013).

Now, we explore what conditions are necessary for a midlevel ethical theory to successfully accompany VSD. The list of desiderata we provide here is, however, not meant to be exhaustive.

\section{Desiderata for a mid-level ethical theory}

1. Explanatory power: the theory should provide us with insight on the purpose and status of morality, and on how principles, rules and rights are related to obligations, and the like (Beauchamp and Childress 2013).

2. Justificatory power: the theory should provide grounds of justification and argumentation for moral claims and considerations. These grounds of justification are necessary to make principled judgments and to legitimize value prioritizations.

3. Simplicity and practicability: the practical requirements of the ethical theory should be simple, i.e.; the practical requirements should not be so demanding that they can only be satisfied by a small number of expert ethicists. We cannot expect everyone on the VSD team to possess (or acquire) adequate knowledge of ethical theory. This raises the question who should conduct the ethical analysis during the VSD process: ought the ethical reflection to be conducted by an ethicist who joins the design team, or by the designers themselves? This is a much-debated topic (Manders-Huits and Zimmer 2009; Van Wynsberghe and Robbins 2013) and goes beyond the scope of this paper. However, it is important that the ethical theory is as simple and practical as possible, so that a broad range of people with interdisciplinary backgrounds can understand and apply the theory to practice.

A mid-level ethical theory that meets the abovementioned desiderata is able to solve the various problems that VSD currently faces, as discussed at the beginning of this paper.

First, a mid-level approach can provide a solution to the problem of making explicit the voice of the researcher at least as well as top-down or bottom-up approaches can. Any given mid-level approach influencing the design process can be named and justified for use in this particular design context. Designers engaging in VSD thus acknowledge they are choosing amongst several different ethical approaches and have chosen one on the basis of normative considerations. However they have not done so randomly or merely as a result of their own personal preferences, but in a way that is grounded in ethical literature and is uniquely appropriate to the domain.

Secondly, mid-level approaches, at least as well as top-down and bottom-up approaches, avoid risking the 
naturalistic fallacy because they have the potential to critically assess current practices, design choices, and arrangements from a moral point of view.

Thirdly, mid-level approaches are able to tell us why certain values should be prioritized over others and how to make value trade-offs in case of value conflicts, better than top-down or bottom-up approaches are able to. That is because the general precepts of top-down approaches are often too indeterminate to provide us with clear action guidance or decision-making content. And bottom-up approaches lack the ability to publically justify value prioritizations or value trade-offs because they obscure the moral deliberation process by denying that moral principles play a role in moral deliberation at all.

Examples of mid-level ethical theories that meet the abovementioned desiderata are the theory of principlism by Beauchamp and Childress (2013), the Capability Approach (Robeyns 2017), or Martin Peterson's geometric account to moral principles (2017). Which of these mid-level theories is best suited to accompany designers in the VSD process is up to further debate.

\section{Concluding remarks}

We believe that VSD practitioners should have the liberty to choose for themselves what ethical theory they make use of to complement the VSD analysis. However, we hope to have convincingly showed that a mid-level ethical theory that meets abovementioned desiderata is most suitable to do so.

Acknowledgements We would like to thank Lily Frank, Anthonie Meijers, Sven Nyholm, and two anonymous reviewers for their valuable comments on earlier drafts of this article.

Open Access This article is distributed under the terms of the Creative Commons Attribution 4.0 International License (http://creativeco mmons.org/licenses/by/4.0/), which permits unrestricted use, distribution, and reproduction in any medium, provided you give appropriate credit to the original author(s) and the source, provide a link to the Creative Commons license, and indicate if changes were made.

\section{References}

Albrechtslund, A. (2007). Ethics and technology design. Ethics and Information Technology, 9(1), 63-72.

Arras, J. (2006) Theory and Bioethics. The stanford encyclopedia of philosophy. Edward N. Zalta (Ed.). Retrieved December 1, 2016 from http://plato.stanford.edu/archives/sum2016/entries/theor y-bioethics/.

Beauchamp, T. L., \& Childress, J. F. (2013). Principles of biomedical ethics. Oxford: Oxford University Press.

Borning, A., \& Muller, M. (2012) Next steps for value sensitive design. In CHI 2012 Proceedings of the SIGCHI conference on human factors in computing systems. Retrieved December 9, 2016, from http://dl.acm.org/citation.cfm?doid=2207676.2208560.

Friedman, B., Kahn, P. H. Jr., Borning, A., \& Huldtgren, A. (2013). Value sensitive design and information systems. In N. Doorn, D. Schuurbiers \& I. van de Poel, M. E. Gorman (Eds.), Early engagement and new technologies: Opening up the laboratory (pp. 55-95). Dordrecht: Springer.

Gordon, J. S., Rauprich, O., \& Vollmann, J. (2011). Applying the fourprinciple approach. Bioethics, 25(6), 293-300.

Manders-Huits, N. (2010). What values in design? The challenge of incorporating moral values into design. Science Engineering Ethics, 17(2), 271-287.

Manders-Huits, N., \& Zimmer, M. (2009). Values and pragmatic action: The challenge of introducing ethical intelligence in technical design communities. International Review of Information Ethics, 10(2), 37-45.

Peterson, M. (2017). The ethics of technology, a geometric analysis of five moral principles. Oxford: Oxford University Press.

Richardson, H. S. (1990) Specifying norms as a way to resolve concrete ethical problems. Philosophy and Public Affairs, 19, 279-310.

Robeyns, I. (2017). Wellbeing, freedom and social justice: The capability approach re-examined. Cambridge: Open Book Publishers.

Ross, W. D. (1930). The right and the good. Oxford: Clarendon Press.

Sterba, J. P. (2005). The triumph of practice over theory in ethics. Oxford: Oxford University Press.

Van den Hoven, J. (2008). Moral methodology and information technology. In K. E. Himma \& H. T. Tavani (Eds.), The handbook of information and computer ethics (pp. 49-68). New Jersey: Wiley.

Van Wynsberghe, A., \& Robbins, S. (2013). Ethicists as designer: A pragmatic approach to ethics in the lab. Science Engineering Ethics, 20(4), 947-961. 